\title{
Automatic Threshold Determination for a Local Approach of Change Detection in Long-Term Signal Recordings
}

\author{
Wassim El Falou, ${ }^{1,2}$ Mohamad Khalil, ${ }^{2}$ Jacques Duchêne, ${ }^{1}$ and David Hewson ${ }^{1}$ \\ ${ }^{1}$ Institut des Sciences et Technologies de l'Information, Université de Technologie de Troyes, France \\ ${ }^{2}$ Faculté de Génie I, Université Libanaise, Tripoli, Lebanon
}

Received 18 October 2006; Revised 26 January 2007; Accepted 27 April 2007

Recommended by Gloria Menegaz

CUSUM (cumulative sum) is a well-known method that can be used to detect changes in a signal when the parameters of this signal are known. This paper presents an adaptation of the CUSUM-based change detection algorithms to long-term signal recordings where the various hypotheses contained in the signal are unknown. The starting point of the work was the dynamic cumulative sum (DCS) algorithm, previously developed for application to long-term electromyography (EMG) recordings. DCS has been improved in two ways. The first was a new procedure to estimate the distribution parameters to ensure the respect of the detectability property. The second was the definition of two separate, automatically determined thresholds. One of them (lower threshold) acted to stop the estimation process, the other one (upper threshold) was applied to the detection function. The automatic determination of the thresholds was based on the Kullback-Leibler distance which gives information about the distance between the detected segments (events). Tests on simulated data demonstrated the efficiency of these improvements of the DCS algorithm.

Copyright (C) 2007 Wassim El Falou et al. This is an open access article distributed under the Creative Commons Attribution License, which permits unrestricted use, distribution, and reproduction in any medium, provided the original work is properly cited.

\section{INTRODUCTION}

Change detection and segmentation are the first steps of many signal processing applications (see, e.g., speech processing [1-4], video tracking [5], ergonomics [6], biomedical applications [7-9], seismic applications [10]). Most detection and segmentation algorithms are based on the theory of statistical detection and hypothesis testing [10-12].

In such an approach, a change occurs when the statistical properties of the signal are modified. Roughly speaking, this can be expressed either by a different distribution function before and after the change time, or by a modification of the parameter value of the same distribution. For the latter case, when the parameter values are a priori known, an efficient algorithm to solve the detection problem is the CUSUM (cumulative sum) algorithm based on the log-likelihood ratio $[10,13]$. CUSUM algorithm is optimal in the sense that it optimizes the worst detection delay when the mean time between false alarms goes to infinity [10].

In many applications, modifications can affect energy, frequency, or both $[14,15]$. Detection of a change in the frequency content can be performed using the CUSUM algorithm applied on the innovation of an $\mathrm{AR}$ (auto regressive) or ARMA (auto regressive moving average) modeling $[4,10]$, the AR (or ARMA) coefficients carrying information about the frequency content of the signal [14].

In usual applications, the parameters corresponding to the segments to be detected are often unknown and other algorithms have to be applied for change detection. Such algorithms can be found in $[9,16]$, based on the computation of a dynamic cumulative sum (DCS) of the likelihood ratio between two locally estimated distributions. These distributions are estimated at each time $t$ using two sliding windows before and after the current time $t$.

In this paper, we propose a modified method of DCS that can be adapted to long duration signals. This modification is achieved on windows length and thresholding. The main application of our study is to detect fatigue of in postural muscles during driving. For that purpose, electromyography (EMG) signals are acquired continuously during a long-term driving task and the first step of the analysis is to detect segments of the signal that contains EMG with a reasonable signal to noise ratio.

The first part of this paper provides an overview of the CUSUM algorithm, focusing on the dynamic cumulative sum to describe its main properties and limits. Then a modified detection algorithm is proposed to go beyond these 


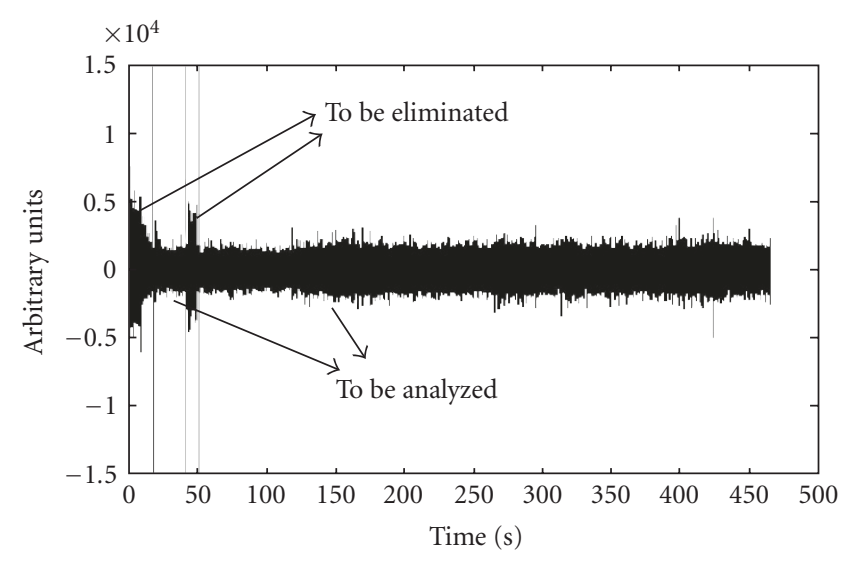

Figure 1: EMG signal and its contents. Contractions have to be detected (segmented) and eliminated. $x$-axis: time in secondS. $y$-axis: arbitrary unit.

limits. An automatic determination of the thresholds is presented in the third part of the paper.

\section{PROBLEM STATEMENT}

The fatigue that can be produced during driving can be detected by studying the EMG signal of the active muscles. In our work, this signal is acquired on the muscles during 2.5 hours of driving, the global aim being to detect the level of the fatigue during driving.

These signals contain a background (low-level) activity corresponding to the postural maintaining (what is the part of interest for the study) as well as high level epochs corresponding to muscle contractions related to voluntary motions. These events have to be eliminated from the signal in order to keep the only muscle activity corresponding to the "resting" state (postural activity: Figure 1).

To eliminate the voluntary contractions from the signal, we developed a new method of detection (MDCS) that can be adapted to long duration signals. After change detection and signal segmentation, the next step (not presented in this paper) would be to compute indices like the median frequency of the resting segments to quantify the fatigue. In this paper we only focus on the first problem of detectionsegmentation.

\section{DCS AS AN EXTENSION OF THE CUSUM ALGORITHM}

\subsection{Overview of the CUSUM algorithm}

Let $\left(x_{1}, x_{2}, \ldots, x_{n}\right)$ be a sequence of observed random variables with conditional probability density $f_{\theta_{0}}\left(x_{k} / x_{k-1}\right.$, $\left.\ldots, x_{1}\right)$ before the change time $t_{0}, \theta_{0}$ being the parameter vector of the segment $S_{0}$ before $t_{0}$, and with conditional probability density $f_{\theta_{1}}\left(x_{k} / x_{k-1}, \ldots, x_{1}\right)$ after this change time, $\theta_{1}$ being the parameter vector of segment $S_{1}$ after $t_{0}$.
Let $S_{1}^{k}$ be the sum of the logarithms of the successive likelihood ratios [10]:

$$
S_{1}^{k}=\sum_{i=1}^{k} s_{i}=\sum_{i=1}^{k} \log \frac{f_{\theta_{1}}\left(x_{i} / x_{i-1}, \ldots, x_{1}\right)}{f_{\theta_{0}}\left(x_{i} / x_{i-1}, \ldots, x_{1}\right)} .
$$

The decision function is defined as

$$
g_{k}=S_{1}^{k}-\min _{1 \leq j \leq k} S_{1}^{j}
$$

and the corresponding stopping time is

$$
t_{s}=\min \left\{k: g_{k} \geq h\right\},
$$

where $h$ is a given threshold.

Given that $E_{\theta_{0}}\left[s_{i}\right]<0$ and $E_{\theta_{1}}\left[s_{j}\right]>0$ (detectability property), an estimated value of change time $t_{0}$ can be obtained by the relation

$$
t_{0}=\max \left\{k: g_{k}=0\right\} .
$$
as $[10]$

The CUSUM algorithm can be written in a recursive way

$$
\begin{gathered}
g_{0}=0, \\
g_{k}=\max \left(0, g_{k-1}+s_{k}\right) .
\end{gathered}
$$

In the case of independent zero mean Gaussian sequences and when the point is to detect a change of variance, the expression of the likelihood ratio becomes [10]:

$$
s_{i}=\frac{1}{2} \ln \frac{\sigma_{0}^{2}}{\sigma_{1}^{2}}+x_{i}^{2}\left(\frac{1}{2 \sigma_{0}^{2}}-\frac{1}{2 \sigma_{1}^{2}}\right),
$$

(Because: $f_{\theta_{0}}\left(x_{i}\right)=\frac{1}{\sqrt{2 \pi} \sigma_{0}} e^{-x_{i}^{2} / 2 \sigma_{0}^{2}} ; f_{\theta_{1}}\left(x_{i}\right)=\frac{1}{\sqrt{2 \pi} \sigma_{1}} e^{-x_{i}^{2} / 2 \sigma_{1}^{2}}$ ).

\subsection{Application after autoregressive modeling}

A signal is AR-modeled if it can be written as

$$
x_{i}=-\sum_{n=1}^{p} a_{n} \cdot x_{i-n}+\varepsilon_{i},
$$

where $\varepsilon_{i}$ are the innovations or prediction errors of the signal (white noise). The terms $a_{i}$ are the coefficients of the model and contain frequency information of the signal. The variance $\sigma_{0}^{2}$ of the innovations gives the energy of the signal. In general, detection cannot be applied on dependent signals. Therefore the change detection algorithm is applied on the sequences of prediction errors deduced from AR (autoregressive) modeling for $S_{0}$ (before change time, $\left.\theta_{0}=\left(a_{1}^{0}, \ldots, a_{p}^{0}, \sigma_{0}^{2}\right)\right)$ and $S_{1}$ (after change time, $\theta_{1}=$ $\left.\left(a_{1}^{1}, \ldots, a_{p}^{1}, \sigma_{1}^{2}\right)\right)[10,14]$ :

$$
s_{i}=\frac{1}{2} \ln \frac{\sigma_{0}^{2}}{\sigma_{1}^{2}}+\frac{\left(\varepsilon_{i}^{0}\right)^{2}}{2 \sigma_{0}^{2}}-\frac{\left(\varepsilon_{i}^{1}\right)^{2}}{2 \sigma_{1}^{2}},
$$

where

$$
\varepsilon_{i}^{l}=x_{i}+\sum_{k=1}^{p} a_{k} x_{i-k} ; \quad l=\{0,1\} .
$$




\subsection{The DCS algorithm}

Many algorithms can be found that detect spectral changes when the parameters are unknown (see, e.g., the Brandt algorithm [10], the divergence Hinkley algorithm [14], DCS algorithm $[9,16])$.

The latter (DCS) was developed for detection of changes in signals of long duration. It was based on local cumulative sums of likelihood ratios computed between two local windows estimated around the current time $t$. The parameters of the two segments, $S_{b}^{t}$ (" $b$ " for "before") and $S_{a}^{t}$ (" $a$ " for "after"), were estimated using two estimation windows $W_{a}$ and $W_{b}$ of identical length $N$ before and after the current time $t$ :

(i) $W_{b}^{t}: x_{i} ; i=\{t-N, \ldots, t-1\}$ used to estimate the parameter $\hat{\theta}_{b}$ of the probability function before the current time $t$,

(ii) $W_{a}^{t}: x_{i} ; i=\{t+1, \ldots, t+N\}$ used to estimate the parameter $\hat{\theta}_{a}$ of the probability function after the current time $t$.

At time $t$, DCS was defined as:

$$
\operatorname{DCS}\left(H_{a}^{t}, H_{b}^{t}\right)=\sum_{j=1}^{t} \log \frac{f_{a}^{j}\left(x_{j}\right)}{f_{b}^{j}\left(x_{j}\right)}=\sum_{j=1}^{t} s_{j} .
$$

When a change occurs at $t_{M}$ it has been demonstrated [9] that DCS reaches a maximum at this time $t_{M}$.

The detection function was expressed as

$$
g(t)=\max _{1 \leq j \leq t}\left[\operatorname{DCS}\left(H_{a}^{j}, H_{b}^{j}\right)\right]-\operatorname{DCS}\left(H_{a}^{t}, H_{b}^{t}\right)
$$

and the stopping time was:

$$
t_{s}=\inf \{t: g(t) \geq h\},
$$

where $h$ was a given threshold.

When applying the DCS algorithm after AR (autoregressive) modeling, a third window $W_{p}^{t}$ was necessary to compute the prediction error after AR parameter estimation.

Figure 2 illustrates the window definition ( $W_{b}^{t}$ for AR parameter $\theta_{b}^{t}$ estimation, $W_{a}^{t}$ for AR parameter $\theta_{a}^{t}$ estimation, $W_{p}^{t}$ for prediction error estimation), the evolution of DCS around the change time $t$, and the corresponding evolution of the decision function.

This change detection method has proved to be efficient when applied to uterine EMG [9] or postural muscle activity [17]. However, some limitations of DCS can be underlined that are related to its use in specific configurations: (i) as the estimation windows are used to estimate locally the distribution parameters before and after the current time $t$, the choice of the window width has a great influence on the detection process; (ii) the detectability property is no longer preserved in the DCS algorithm. Therefore detection fails when the two distributions are very close together (see Figure 3). In fact, the detection function stabilizes after $N$ points beyond the change time without reaching the threshold.

Based on the same basic concept, a modified algorithm was developed to overcome these problems and to ensure the detectability property.

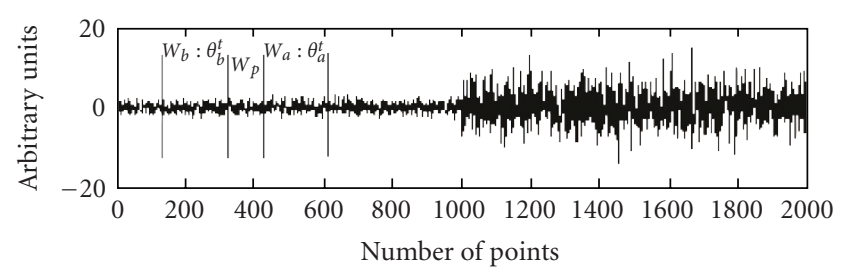

(a)

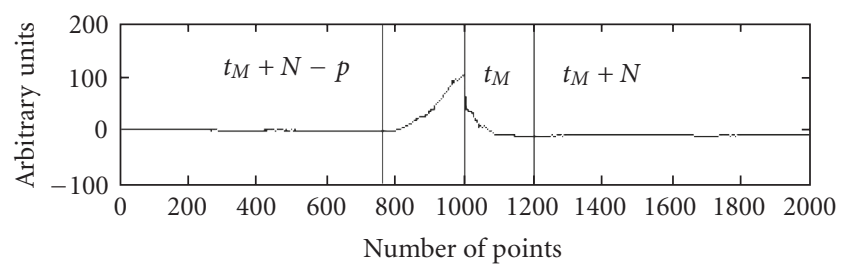

(b)

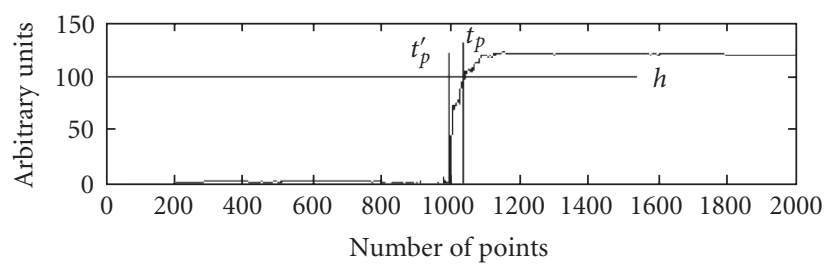

(c)

FIGURE 2: Upper tracing: position of the estimation and prediction windows. Middle tracing: evolution of DCS around the change time $t_{M}$. Lower tracing: detection function. For all tracings, $x$-axis: number of points, $y$-axis: arbitrary units.

\section{THE MODIFIED DYNAMIC CUMULATIVE SUM (MDCS) ALGORITHM}

\subsection{Variable window width}

The algorithm is still based on two sliding windows $W_{b}^{t}$ and $W_{a}^{t}$ that are used to estimate $\theta_{b}^{t}$ and $\theta_{a}^{t}$ at each time $t$. As for DCS, $W_{a}^{t}$ has a constant length $N$, but $W_{b}^{t}$ now includes all samples from 1 to $t-1$. Hence, when both windows correspond to the same distribution (no change in the segment), the parameter estimation is always better for $W_{b}^{t}$ than for $W_{a}^{t}$, leading to $E_{\theta_{0}}\left[s_{i}\right]<0$.

$\theta_{b}^{t}$ and $\theta_{a}^{t}$ are estimated using these new windows:

$$
\begin{gathered}
W_{b}^{t}: 1 \cdots t-1 \longrightarrow \theta_{b}^{t}, \\
W_{a}^{t}: t+1 \cdots t+N \longrightarrow \theta_{a}^{t} .
\end{gathered}
$$

The definitions of the log-likelihood ratios, the cumulative sum, and the detection function remain the same as for DCS. In addition, when the signal samples are dependent, it is still possible to perform AR modeling and to introduce an intermediate prediction window $W_{p}^{t}$.

Figure 4 illustrates this new approach. MDCS is now decreasing before the change time and continuously increasing after that, if the process is not stopped by a threshold crossing. 


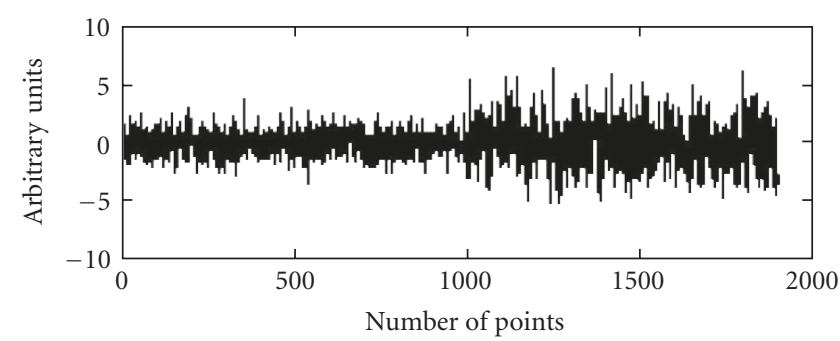

(a)

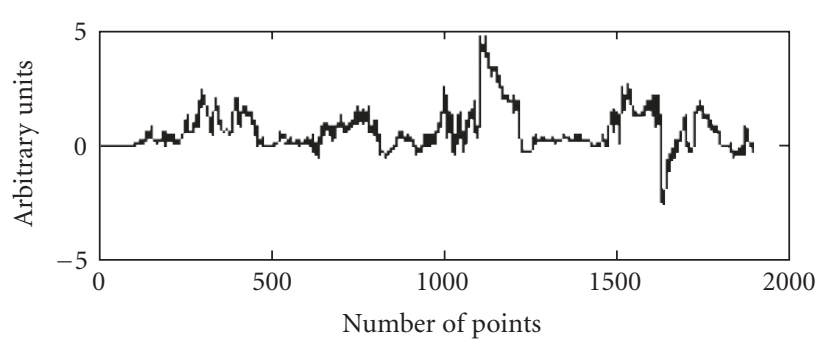

(b)

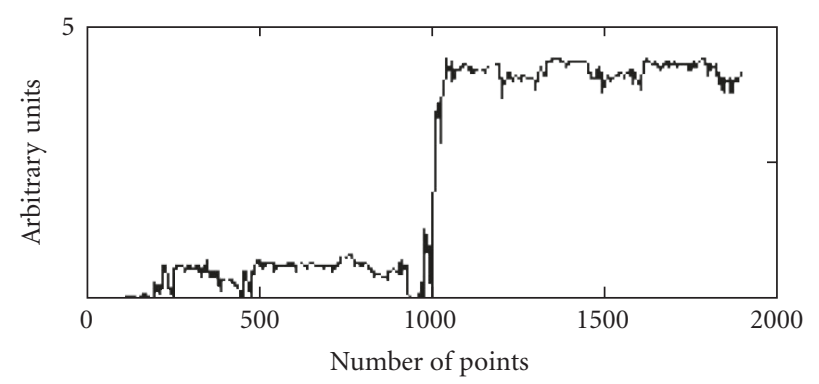

(c)

FIgURE 3: An illustration of a change that was not detected by the DCS algorithm. Upper tracing: signal segment. Middle tracing: DCS evolution. Lower tracing: detection function evolution. For all tracings, $x$-axis: number of points, $y$-axis: arbitrary units.

\subsection{Double thresholding}

One of DCS drawbacks was the fact that, between the change time and the stopping time, $W_{b}^{t}$ kept increasing, hence including samples taken after the change time to update $\theta_{0}$ estimates. To solve this problem, the idea was to apply two thresholds $\left(h_{L}\right.$ and $\left.h_{H}\right)$ to the detection function:

(i) the lower threshold $h_{L}$ stops $\theta_{0}$ estimate updating,

(ii) the higher threshold detects the change $h_{H}$.

This double thresholding allows a limitation in the bias of $\theta_{0}$ estimation without increasing the false alarm rate, as was the case before when a threshold that was too low was applied to the detection function.

\section{AUTOMATIC CHOICE OF THE THRESHOLDS}

One of the most crucial issues in change detection is the choice of the detection threshold $h$. It mainly depends on the signal characteristics and is generally adjusted by expe-

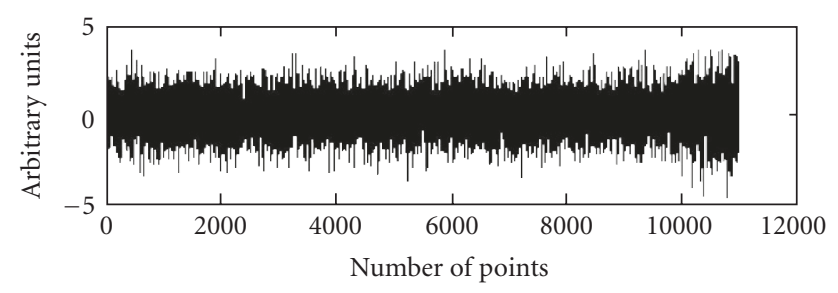

(a)

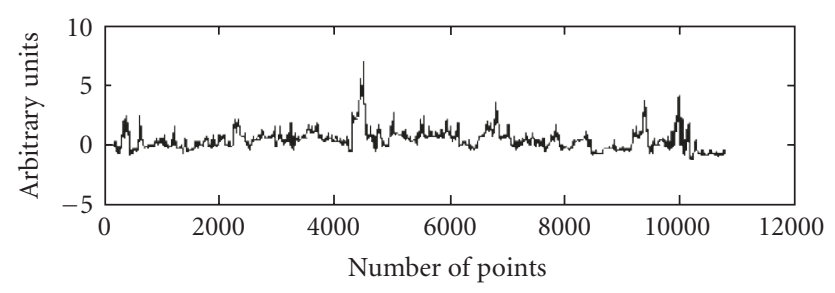

(b)

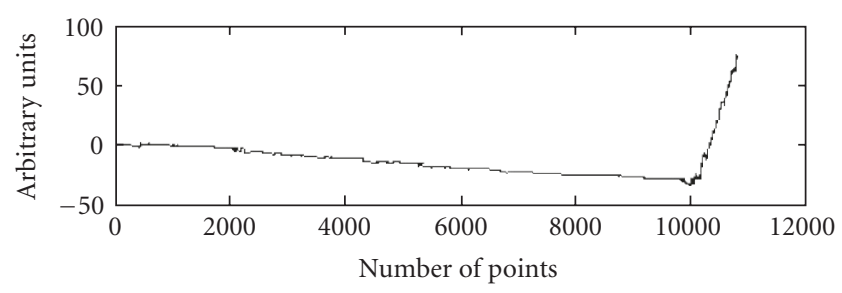

(c)

Figure 4: (a) Simulated signal containing a change in variance (from 1 to 2) at point 10000. (b) Evolution of DCS before and after the change time (change not detected). (c) Evolution of MDCS before and after the change time (change detected). For all tracings, $x$-axis: number of points, $y$-axis: arbitrary units.

rience or by using a training set of data. Methodologies can be found in the literature to choose the threshold according to the probability of false alarm, and the mean time between false alarms $[10,18]$. However, the formulation is asymptotic and difficult to apply in practical use.

In case of a CUSUM algorithm, a very useful factor to choose the threshold $h$ is the Kullback-Leibler distance between two probability densities $f_{\theta_{0}}$ and $f_{\theta_{1}}$ of a random variable $x$, defined as

$$
K\left(\theta_{0}, \theta_{1}\right)=\int \operatorname{Ln} \frac{f_{\theta_{0}}(x)}{f_{\theta_{1}}(x)} f_{\theta_{0}}(x) d x .
$$

The Kullback-Leibler distance can be considered as a distance between these two probability densities. In addition, it is known [10] that the delay for detection is inversely proportional to the Kullback-Leibler distance. If $h$ is the threshold used in the detection algorithm, the relationship between $h$ and the Kullback-Leibler distance can be expressed as

$$
E_{\theta_{1}}(s)=K\left(\theta_{1}, \theta_{0}\right)=\frac{h}{\tau},
$$

where $\tau$ is the mean delay for detection. Hence the KullbackLeibler distance can be used to choose the threshold $h$. 


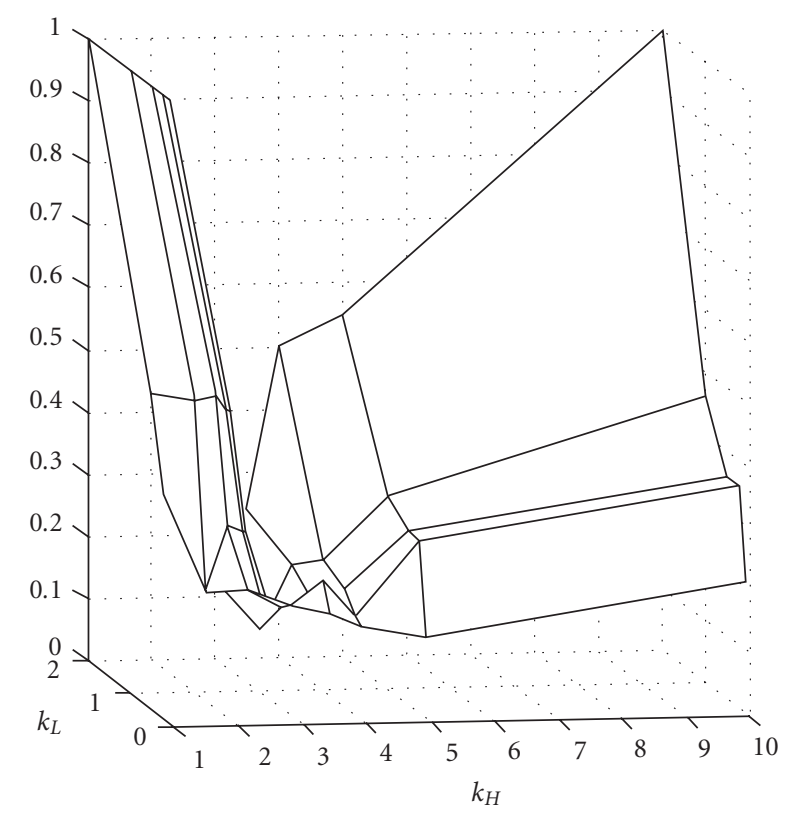

FIGURE 5: Variation of the segmentation error with the low and high threshold values. Thresholds are indicated as the factors $k_{L}$ ( $y$-axis) and $k_{H}$ ( $x$-axis) to apply to the mean square value of the KullbackLeibler distance $\mathrm{MS}_{\mathrm{KL}}$.

From (16) we can write $h \approx M \cdot K\left(\theta_{1}, \theta_{0}\right)$ where $M$ is the number of points after time changes. So we can use an estimation of the Kullback-Leibler distance to calculate the threshold $h$.

Now considering two AR models $\theta_{0}=\left(a_{1}^{0}, \ldots, a_{p}^{0}, \sigma_{0}^{2}\right)$ and $\theta_{1}=\left(a_{1}^{1}, \ldots, a_{p}^{1}, \sigma_{1}^{2}\right)$, the Kullback-Leibler distance between $\theta_{1}$ and $\theta_{0}$ can be expressed as [6]

$$
K\left(\theta_{1}, \theta_{0}\right)=-\frac{1}{2}-\frac{1}{2} \ln \frac{\sigma_{1}^{2}}{\sigma_{0}^{2}}+\frac{1}{2} \frac{\sigma_{1}^{2}}{\sigma_{0}^{2}}\left[1+\sum_{k=1}^{\infty}\left(c_{k}^{0 / 1}\right)^{2}\right],
$$

where the coefficients $c_{k}^{0 / 1}$ are the coefficients of the following Taylor expansion:

$$
\frac{A_{0}(z)}{A_{1}(z)}=1+\sum_{k=1}^{\infty} c_{k}^{0 / 1} z^{-k}
$$

The following steps are proposed to choose the threshold $h$ automatically.

(1) The signal is first divided into successive segments of equal length $N$.

(2) The AR model $\theta=\left(a_{1}, \ldots, a_{p}, \sigma^{2}\right)$ is estimated for each segment.

(3) Then the Kullback-Leibler distance is computed between each pair of successive segments, leading to a sequence of values that are thus sorted in ascendant order.

(4) The sequence is limited to the lowest $90 \%$ of values in order to suppress the influence of any possible very large value.
(5) The mean square value $\mathrm{MS}_{\mathrm{KL}}$ of the remaining distribution of the Kullback-Leibler distances is then computed, providing the low and high thresholds $h_{L}=$ $N \cdot k_{L} \cdot \mathrm{MS}_{\mathrm{KL}}$ and $h_{H}=N \cdot k_{H} \cdot \mathrm{MS}_{\mathrm{KL}}, N$ being the window width.

The determination of $k_{L}$ and $k_{H}$ was performed by simulation with the same reference set as that used to build the ROC curves in the previous paragraph. Segmentation was achieved with successive values of $k_{L}$ and $k_{H}$ and the number of nondetection and false alarms counted.

Figure 5 shows the variation of the segmentation error (sum of nondetections and false alarms) with respect to both thresholds using the simulation data. The surface presents a minimum at $k_{L}=1$ and $k_{H}=3$.

\section{RESULTS AND DISCUSSION}

This method was first tested on simulated signals generated by concatenating segments of random noise filtered at different frequency bands, then to electromyographic recordings.

As an illustration, the segmentation was applied to electromyographic signals recorded during a long term $(2 \mathrm{~h} 30)$ experiment assessing the comfort of car seats through a measure of local muscular fatigue. Each experiment was divided into 7 phases lasting from 10 minutes to 30 minutes. Figure 6 shows one of those phases after MDCS segmentation (25 segments).

This new technique of windowing-double thresholds decreases the probability of false alarm especially in the electromyography signals which are long duration signals. This is coming from the fact that the detection function $g(t)$ rises to the second threshold only when a real change occurs. Furthermore, The Kullback-Leibler distance is used to determine these thresholds automatically because the characteristics of the electromyography signals change from person to another and depend on many other parameters. Finally, it is important to notice that this method can be applied to whatever kind of signals presenting changes in frequency or amplitude.

Both methods (DCS and MDCS) were tested on simulated data made of 1000 segments of white noise with a variance change from 1 to 2 and 1000 segments without a change. To compare the results, we chose the ROC curves (receiver operating characteristics) that plot the probability of detection with respect to the probability of false alarms. In general, higher is the curve, better are the results. Figure 7 clearly shows how the modified algorithm improves the overall detection quality.

In these curves presented on Figure 7, we can see that if we need a detection probability equal to 0.9 , the false alarm probability given by the DCS algorithm is about 0.1 but it is less than 0.02 for the MDCS method. MDCS decreases the probability of false alarm for a given detection probability.

\section{CONCLUSION}

The local approach of change detection allows a local estimation of the distribution parameters before and after the current time $t$. A change is detected in the same way as 


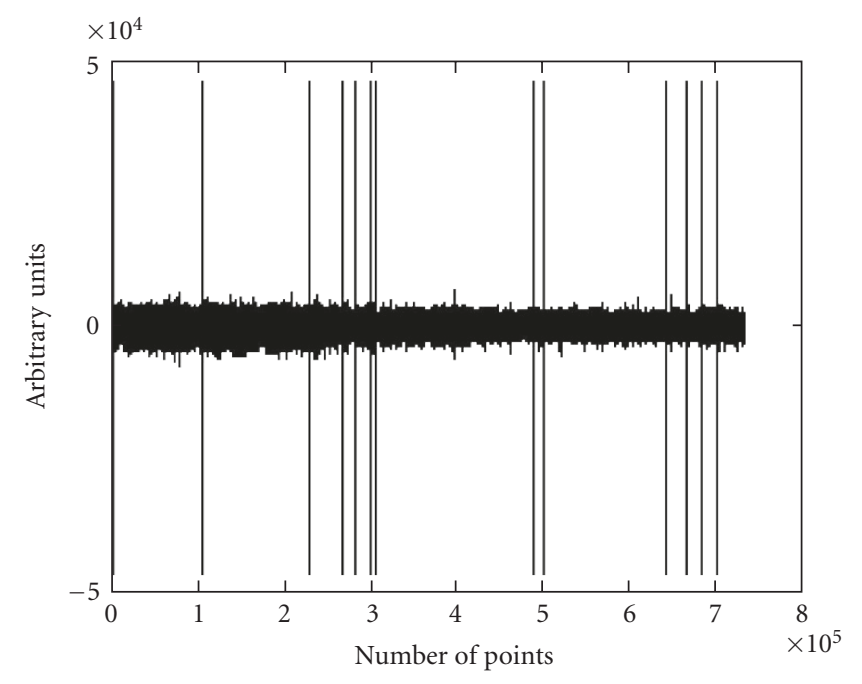

(a)

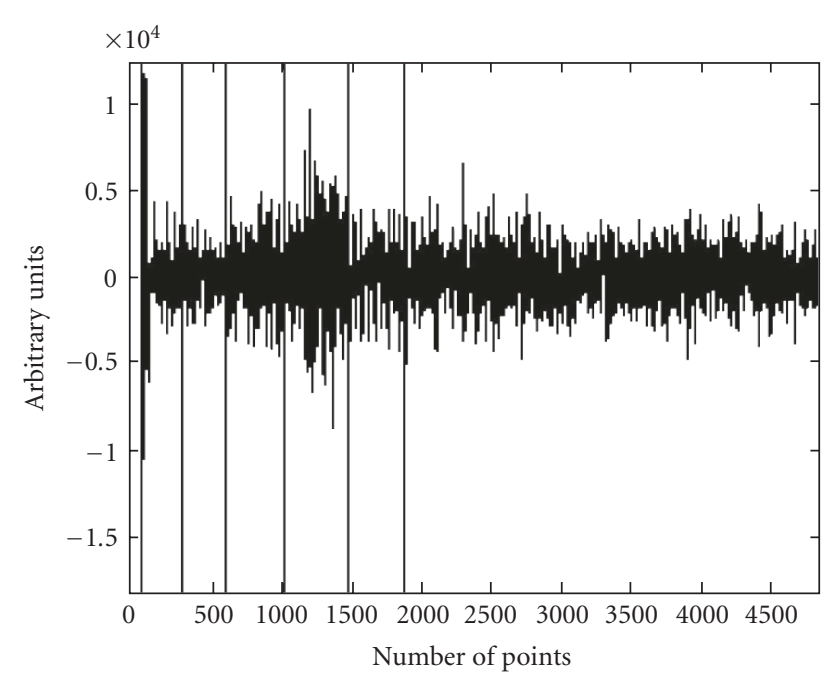

(b)

FIGURE 6: Application of MDCS on a real signal. (a) a 15-minute recording epoch, (b) zoom at the beginning of the signal. This figure shows the detection points. $x$-axis: number of points, $y$-axis: arbitrary units.

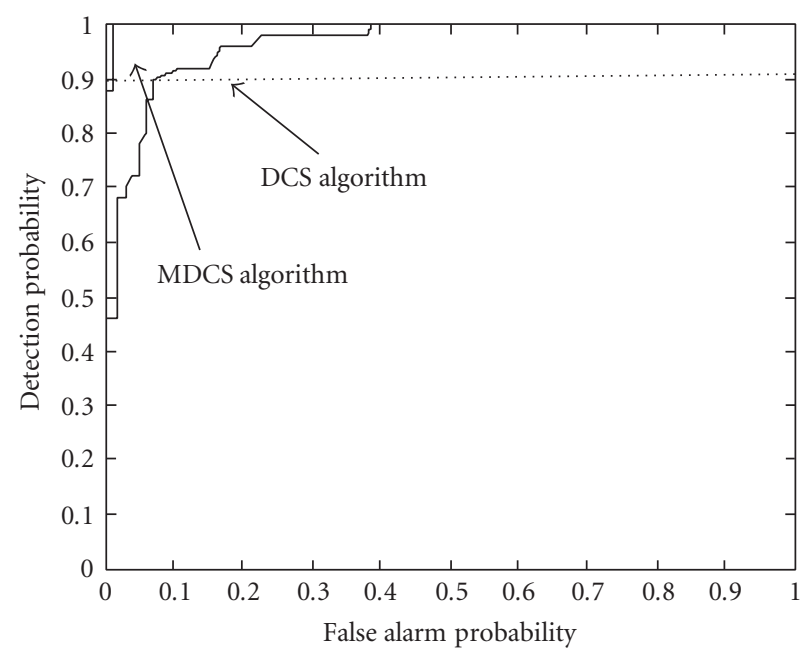

FIgURE 7: Comparison of DCS and MDCS methods by ROC curves computed from simulated data. $x$-axis: false alarm probability, $y$ axis: detection probability.

for the classical CUSUM approach after parameter estimation. A first algorithm (DCS) had been successfully tested on long term recordings related to biomedical signals. However, DCS presented some limitations in its ability to detect slow changes, the main of them being that it did not respect the detectability property. In addition, the threshold of the detection function had to be chosen by expertise or by using reference data sets. The modified algorithm overcomes these problems by a restriction of the estimation window for the segment $S_{0}$ (before change point) using a low threshold that is distinct from the detection function threshold itself. In addition, these thresholds are learned automatically by using the Kullback-Leibler distance. As a consequence, MDCS becomes an offline algorithm if applied extensively to each recording to be segmented, since the Kullback-Leibler distance distribution must be computed first for each new recording. However, it seems wise to imagine that the same thresholds could be applied to a class of similar signals such as electromyograms recorded on various muscles and various subjects during the same experimental protocol. Nevertheless this point has yet to be demonstrated.

\section{REFERENCES}

[1] R. A. Obrecht, "A new statistical approach for the automatic segmentation of continuous speech signals," IEEE Transactions on Acoustics, Speech, and Signal Processing, vol. 36, no. 1, pp. 29-40, 1988.

[2] R. A. Obrecht, B. Jacob, and N. Parlangeau, "Audio visual speech recognition and segmental master slave HMM," in Proceedings of ESCA Workshop on Audio-Visual Speech Processing (AVSP '97), pp. 49-52, Rhodes, Greece, September 1997.

[3] R. A. Obrecht and H. Y. Su, "Three acoustic label lings for phoneme based continuous speech recognition," in Proceedings of the 7th FASE Symposium (SPEECH '88), pp. 943-950, Edinburgh, Scotland, August 1988.

[4] S. R. Turajlić and Z. M. Šarić, "Sequential speech segmentation based on the spectral arma transition measure," Circuits, Systems, and Signal Processing, vol. 15, no. 1, pp. 71-92, 1996.

[5] J. Calic, "Experimental framework for TRECVID 2006," University of Bristol, http://www-nlpir.nist.gov/projects/trecvid/.

[6] J. Duchêne and Th. Lamotte, "Surface electromyography analysis in long-term recordings: application to head rest comfort in cars," Ergonomics, vol. 44, no. 3, pp. 313-327, 2001.

[7] M. Chendeb, M. Khalil, and J. Duchêne, "The use of wavelet packets for event detection," in Proceedings of the 13th European Signal Processing Conference (EUSIPCO '05), Antalya, Turkey, September 2005. 
[8] M. Chendeb, M. Khalil, and J. Duchêne, "Methodology of wavelet packet selection for event detection," Signal Processing, vol. 86, no. 12, pp. 3826-3841, 2006.

[9] M. Khalil and J. Duchêne, "Uterine EMG analysis: a dynamic approach for change detection and classification," IEEE Transactions on Biomedical Engineering, vol. 47, no. 6, pp. 748-756, 2000.

[10] M. Basseville and I. Nikiforov, Detection of Abrupt Changes: Theory and Application, Prentice-Hall, Englewood Cliffs, NJ, USA, 1993.

[11] S. Aivazian, I. Enukov, and L. Mechalkine, Eléments de Modélisation et Traitement Primaire des Données, Mir, Moscow, Russia, 1986.

[12] A. Borovkov, Statistique Mathématique, Mir, Moscow, Russia, 1987.

[13] E. S. Page, "Continuous inspection schemes," Biometrika, vol. 41, no. 1-2, pp. 100-115, 1954.

[14] M. Basseville and A. Benveniste, "Sequential detection of abrupt changes in spectral characteristics of digital signals," IEEE Transactions on Information Theory, vol. 29, no. 5, pp. 709-724, 1983.

[15] M. Khalil and J. Duchêne, "Detection and classification of multiple events in piecewise stationary signals: comparison between autoregressive and multiscale approaches," Signal Processing, vol. 75, no. 3, pp. 239-251, 1999.

[16] M. Khalil, "Une approche de la détection et de la classification dans les signaux non stationnaires. Application à l'EMG utérin," Thèse de Doctorat, l'Université de Technologie de Troyes, Troyes, France, 1999.

[17] W. El Falou, J. Duchêne, M. Khalil, and Y. Langeron, "Segmentation avec rejet de signaux EMG posturaux par une méthode locale," in Proceedings of the 18th Symposium on Signal and Image Processing (GRETSI '01), pp. 536-748, Toulouse, France, September 2001.

[18] M. I. Baron, "Nonparametric adaptive change-point estimation and on-line detection," Sequential Analysis, vol. 19, no. 12, pp. 1-23, 2000.

Wassim El Falou was born in Lebanon in 1975. He received the Diploma in engineering (electrical-electronics) from the Lebanese University, and a M.S in mathematical modelling from Saint Joseph and Lebanese Universities. He received his Ph.D. degree in 2002 from the University of Technology of Troyes, in surface EMG signal processing. He is currently teaching at several universities in Lebanon, including the

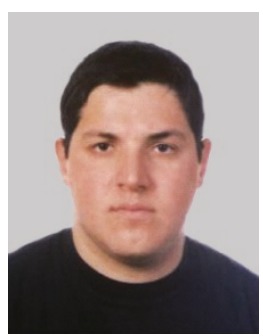
Lebanese University. His main research interests are embedded systems design, signal processing, classification methods, and voice recognition.

Mohamad Khalil was born in Akkar Atika, in Lebanon, in 1973. He obtained the Engineering degree in electrical and electricity from the Faculty of Engineering, Lebanese University, Tripoli, Lebanon, in 1995. He received the D.E.A degree in biomedical engineering from the University of Technology of Compiegne (UTC) in France, in 1996. He received his Ph.D. degree from the University of Technology of Troyes in France, in

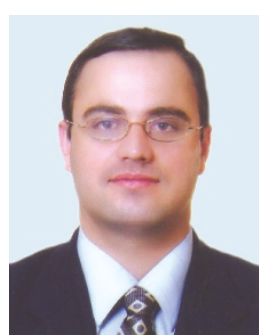
1999. He received his HDR (Habilitation a diriger des recherches) degree from UTC in 2006. He is currently a Researcher at several universities in Lebanon including the Lebanese University. His current interests are the signal and image processing problems: detection, classification, analysis, representation, and modeling of nonstationary signals, with application to biomedical signals and images.

Jacques Duchêne received the Engineer degree in electronics from the Ecole Supérieure d'Electricité, France in 1973, and the doctorat d'état in sciences in 1983. He joined the University of Technology of Troyes in 1994, where he is currently in charge of the Charles Delaunay Institute of Research. His main research interests are signal processing, pattern recognition and classification. He now focuses on signal

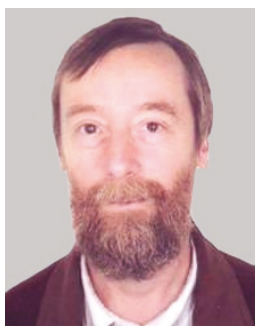
segmentation as well as signal decomposition. The main application fields in biomedical engineering are ergonomics (comfort in cars), biomedical monitoring (quality of balance for elderly), and EMG characterization and modelling (frequency parameters, conduction velocity distribution).

David Hewson received the BPhEd and MPhEd degrees from the University of Otago in New Zealand in 1990 and 1993 and a Ph.D. degree from the University of Auckland in 2000. He worked as a Research Physiologist for the Royal New Zealand Air Force between 1994 and 2000, before undertaking two years of postdoctoral study at the University of Technology of Troyes in France. He is now an Associate Professor at

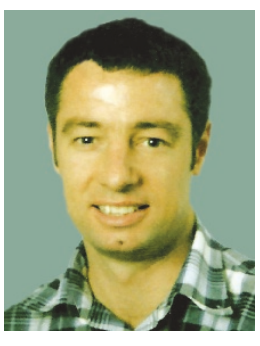
the University of Technology of Troyes. His research interests are ergonomic and clinical applications of surface electromyography. 